\title{
Zwischen Theorie und Praxis
}

\author{
Christina Beck und Julia Wandt
}

Die Rahmenbedingungen, unter denen Wissenschaftskommunikatoren heute arbeiten, haben sich fundamental geändert. Seit Jahren müssen Hochschulen und Forschungseinrichtungen mit einer großen Diversifizierung ihrer Arbeit und ihrer Zielgruppen in der Kommunikation umgehen. Dazu gehören eine gestiegene Anzahl und Vielfalt der Kommunikationskanäle (neue Kanäle kommen hinzu, bestehende fallen nicht weg), aber ebenso der Aufgabenbereiche.

Eine vergleichsweise neue wie unerlässliche Entwicklung ist, dass Hochschulen und Forschungseinrichtungen auch die politische Kommunikation als eine strategische Aufgabe ansehen. Alle Institutionen müssen sich in Wettbewerben wie zum Beispiel der Exzellenzinitiative des Bundes und der Länder und deren Nachfolgeprogramm, der Exzellenzstrategie, oder aber im Rahmen des Pakts für Forschung und Innovation positionieren und die Leistungen ihrer Einrichtung sichtbar machen. Wissenschaftskommunikation selbst ist dabei zum strategischen Erfolgsfaktor geworden.

Eine vom Bundesverband Hochschulkommunikation initiierte Studie des Instituts für Germanistik am Karlsruher Institut für Technologie (KIT)

\author{
C. Beck $(\bowtie)$ \\ Max-Planck-Gesellschaft, München, Deutschland \\ E-Mail: beck@gv.mpg.de \\ J. Wandt \\ Universität Konstanz, Konstanz, Deutschland \\ E-Mail: julia.wandt@uni-konstanz.de
}


unter dem Titel „Hochschulkommunikation erforschen“ (Leßmöllmann et al. 2017; Schwetje et al. 2017) bestätigt die extrem große Aufgabenvielfalt und konstatiert zugleich, dass Kommunikatorinnen und Kommunikatoren innerhalb ihrer Einrichtungen sehr vielfältige und unterschiedliche Rollen einnehmen: vom „Übersetzer“, „Mediator", „Dienstleister“ über „Administrator" bis zu „Popularisierer“ und „Gatekeeper“ (Schwetje et al. 2017, S. 33 ff.). Die Rollenbilder sind trotz großer Heterogenität der Hochschultypen teilweise sehr homogen. Ungeachtet dessen steckt der Alltag von Wissenschaftskommunikatoren nach wie vor in systemischen Zwängen, die sich oft nur schwer überwinden lassen.

\section{Geschätztes Expertenwissen}

„Battle for Truth“ („Kampf um die Wahrheit“) - unter diese Überschrift haben die Autoren des Edelman Trust Barometer (2018) ihre Befunde gestellt. 33.000 Personen aus 28 Ländern nahmen an der Umfrage teil. Danach steigt weltweit die Sorge, dass Falschinformationen als Waffe eingesetzt werden; fast 7 von 10 Personen teilen diese Angst. Mehr als die Hälfte der Befragten findet es immer schwieriger, guten Journalismus von Gerüchten und Falschinformationen zu unterscheiden. Die Schwierigkeit, wahr und unwahr voneinander zu trennen, führt zu einem Vertrauensverlust in Institutionen. Wem kann man noch trauen? Hier offenbart das Edelman Trust Barometer einen weiteren wichtigen Befund: Der Experte gilt nach wie vor als sehr glaubwürdig. Mit 61 Prozent rangiert der akademische Experte bei der Frage, wem vertraut wird, sogar deutlich vor den eigenen Peers, die mit 54 Prozent ein "all-time low", also den niedrigsten Wert seit Jahren erreichen (Edelman Trust Barometer 2018).

Was folgt daraus für die Wissenschaftskommunikation? Das größte Potenzial liegt offenbar nach wie vor in der Kommunikation durch die Wissenschaftlerin oder den Wissenschaftler selbst. Doch genau hier stößt Wissenschaftskommunikation auf nicht zu unterschätzende Hürden. Da wäre zum einen die Kommunikationsfähigkeit. Im 21. Jahrhundert ist damit schon längst nicht mehr nur die Frage verbunden, wer sich als Vortragender vor einem Laienpublikum oder aber als Autor eines populärwissenschaftlichen Beitrags eignet. Es geht nicht nur darum, die Komplexität des eigenen Forschungsfeldes so weit herunterzubrechen, dass Laien dem folgen können. Angesichts der schieren Informationsflut auf den unterschiedlichsten Kanälen und der Vernetzung durch neue Medien wird Aufmerksamkeit zum knappsten Gut. Nicht die Information oder der Zugang zu 
Information ist mehr begrenzt, sondern die Möglichkeit, diese aufzunehmen und zu verarbeiten. Aufmerksamkeit wird zur Währung erfolgreicher Kommunikation.

Was bedeutet das? Inhalte müssen nicht nur vereinfacht, sie müssen auch in möglichst kurzen Einheiten vermittelt werden, die vom Nutzer einfach konsumiert werden können - getreu dem Motto „weniger ist mehr“. Auf die Spitze treiben das die sozialen Medien: 280 Zeichen bei Twitter, 30 Sekunden-Clips bei Facebook, eine Grafik bei Instagram. Kommunikationsverantwortliche ernten in der Regeln Kopfschütteln bei ihren Wissenschaftlern: So ließe sich komplexe Wissenschaft nicht vermitteln, der Tiefgang fehle und es drohe die Gefahr, dass die Botschaften verknappt und unsachlich zugespitzt werden. Die Politik wiederum fordert: Wissenschaft müsse aus dem Elfenbeinturm heraus, es müssten Formate und Medien bedient werden, wo sich Gesellschaft und Wissenschaft auf Augenhöhe begegnen können, und das „natürlich Web-2.0-kompatibel“ und mit dem „Handwerk der Reduktion“.

Bestes Beispiel dafür: der Wetterbericht. Das ist eine gute Plattform, um Zuschauerinnen und Zuschauer darüber zu informieren, was der Klimawandel bewirkt. In einem Beitrag vom 3. September 2018 zitiert die Süddeutsche Zeitung den Kommunikationsforscher Ed Maibach von der George Mason University, der Wettermoderatoren dazu ermutigt, Hintergrundinformationen über Durchschnittstemperaturen, die Zunahme von Waldbränden oder den Rückgang der Regenmengen in die Berichte aufzunehmen: "Zehn Sekunden, mehr braucht man dazu gar nicht" (Schrader 2018). Kurz, prägnant und eingängig - so kann Wissenschaftskommunikation also auch sein.

Soziale Medien wie Twitter funktionieren ähnlich: So kommentiert der Präsident des Deutschen Instituts für Wirtschaftsforschung (DIW), Marcel Fratscher, seit fünf Jahren auf Twitter u. a. die Entscheidung der US-Notenbank Fed, den Leitzins ein weiteres Mal zu erhöhen, oder verweist auf den neuen DIW-Report, der zeigt, wie sehr Deutschland zwischen 2011 und 2016 von der Zuwanderung aus dem europäischen Ausland profitiert hat. Auch der Klimaforscher Stefan Rahmstorf vom Potsdamer Institut für Klimafolgenforschung (PIK) kommentiert in seinem Twitter-Account aktuelle Studien zum Klimawandel, die Berichterstattung zur Klimaforschung in den Medien oder politische Ereignisse wie den Klimagipfel im Dezember 2018 in Polen.

Die Follower-Zahlen muten noch bescheiden an - Fratscher hat 14.000, Rahmstorf 21.000 Follower (Stand: Januar 2019). Aber die Kollegen im angelsächsischen Raum zeigen, was möglich ist: So postet der Evolutionsbiologe Richard Dawkins von der Universität Oxford seit zehn Jahren auf 
Twitter und erreicht dort über 2,7 Millionen Follower (Stand: Januar 2019), dem Physiker Brian Green von der Columbia University, Mitbegründer der String-Theorie, folgen dort eine Million Menschen, und dem Kognitionsforscher Steven Pinker von der Harvard University immer noch fast eine halbe Millionen (alle drei sind jedoch auch bekannte Sachbuchautoren).

Die überwiegende Mehrheit der Wissenschaftler in Deutschland steht dem 280-Zeichen-Kanal jedoch weiterhin skeptisch gegenüber, sie können keinen Nutzen für sich darin erkennen. Vielen fehlt aber auch schlicht die Zeit, um einen solchen Kanal zu betreiben. Und so twittern derzeit vor allem die Kommunikationsabteilungen der Hochschulen und Forschungseinrichtungen (@maxplanckpress hat 141.000 Follower, große Universitäten wie@UniCologne 38.300 oder @TU_Muenchen 24.800; Stand: Januar 2019). Als „institutionelle Kanäle“ erreichen sie in weiten Teilen jene, die schon Teil der Wissenschaftsgemeinde sind oder es werden wollen. Und ihnen fehlt, was einen Social-Media-Kanal eigentlich ausmacht: die Authentizität, die eine einzelne Person besitzt, und damit verbunden die Möglichkeit zu spontaner Meinungsäußerung. Niemand kann besser über ein wissenschaftliches Thema kommunizieren und sich klarer positionieren als die Wissenschaftlerin, der Wissenschaftler selbst.

Für viele Wissenschaftlerinnen und Wissenschaftler bleibt, wenn kommuniziert werden soll, der Namensbeitrag in einer der großen überregionalen Zeitungen nach wie vor das Wunschziel - denn er sichert, so zumindest die Erwartung, Sichtbarkeit bei Entscheidungsträgern und bei der eigenen Community. Dass es in dem Fall ein Elitendialog bleibt - die Überregionalen erreichen mit ihren Print-Produkten nicht viel mehr als fünf Prozent der deutschen Bevölkerung - und die Sichtbarkeit auf anderen Kanälen viel größer sein kann, wird dabei gerne übersehen.

\section{Publikationsflut in der Wissenschaft}

Das Gros der Kommunikationsarbeit liegt also bei den Kommunikationsabteilungen und die schlagen sich mit einer Flut von Fachartikeln herum, die ihnen ihre Wissenschaftler zuschicken verbunden mit der Bitte, diesen mit einer Pressemeldung zu mehr Aufmerksamkeit zu verhelfen. Pro Jahr erscheinen inzwischen weltweit mehr als zwei Millionen Fachartikel. Das Peer-Review-System stehe am Rand des Kollapses, da es die Fülle an Publikationen nicht mehr angemessen verarbeiten könne, kritisierte die renommierte österreichische Wissenschaftsforscherin Helga Nowotny in einem Interview mit der Wochenzeitung DIE ZEIT im August 2018 (Schnabel 
und Spiewak 2. August 2018). Und Philip G. Altbach und Hans de Wit vom Center for International Higher Education am Boston College in den USA forderten in einem Beitrag für University World News eine drastische Reduzierung der Zahl der Publikationen: Tatsächlich könnte man auf mehr als die Hälfte der Forschungsartikel verzichten („... more than half of current so-called research articles could be eliminated"; Altbach und de Wit 7. September 2018).

Der Präsident der Deutschen Forschungsgemeinschaft (DFG), Peter Strohschneider, hat es in einem Interview 2017 treffend beschrieben: „Im Wissenschaftssystem gibt es die Gratifikationen nicht für Kommunikation, sondern für Papers. Darunter leiden alle anderen Funktionen, etwa die akademische Lehre, darunter leiden etwa Transferbereiche und darunter leidet auch die Wissenschaftskommunikation." (Wissenschaft kommuniziert 7. Februar 2017). Und sie leidet gleich in zweierlei Hinsicht: Nicht nur dass die Forschenden keinen Anreiz haben, Kommunikationsleistungen außerhalb des Wissenschaftssystems zu erbringen. Wissenschaftskommunikatoren müssen auch selektieren zwischen dem, was nur für die Wissenschaftsgemeinde, und dem, was für ein nichtwissenschaftliches Publikum von Interesse sein könnte. Nicht immer machen sie sich mit einer solchen Selektion beliebt.

Die vielen Fachartikel müssen zudem nicht nur gelesen, sondern durch andere Forscher auch überprüft werden. Die in einem Fachartikel publizierten Daten stellen per se noch kein neues Wissen dar, sondern werden damit erst zur Diskussion innerhalb der Wissenschaftsgemeinde gestellt. Deshalb ist es eigentlich Unfug, wenn Kommunikationsabteilungen - und übrigens ebenso die Verlage - einzelne Fachartikel (gerne mit hohem Impact Factor als Qualitätsausweis) zum Gegenstand einer Pressemeldung machen. Zum Schluss werden manchmal dann auch Beiträge in den Medien „hochgejazzt“, die sich später als falsch - oder schlimmer noch: als gefälscht herausstellen.

Wissenschaft ist hochgradig kompetitiv - es gibt einen ausgeprägten Wettbewerb um Mittel, Stellen und Reputation. Damit steigt der Druck für die einzelne Wissenschaftlerin bzw. den einzelnen Wissenschaftler, die Relevanz der eigenen Arbeit innerhalb und außerhalb der Wissenschaft zu belegen oder zu konstruieren. Darin liegt ein nicht zu unterschätzendes Glaubwürdigkeitsrisiko für die Wissenschaft. Die Arbeit von Kommunikationsabteilungen von Hochschulen und Forschungseinrichtungen ist zweifelsohne interessengelenkt. Trotzdem sollte sie sich nicht so leicht vor den PR-Karren spannen lassen. Nicht zu jedem Nature-oder Science-Paper braucht es eine Pressemeldung, nicht jedes dieser wissenschaftlichen Puzzlesteine ist für die Welt außerhalb der Wissenschaft von Interesse. 
Wissenschaftskommunikation sollte außerdem, wenn sie überzeugen will, Widersprüche aufzeigen und dabei gegebenenfalls auch auf die Ergebnisse anderer Forschungsgruppen verweisen. Sie muss die wissenschaftliche Debatte transparenter machen und die Grenzen wissenschaftlicher Erkenntnisse aufzeigen. Und sie sollte nicht nur - wie im Journalismus üblich - nach dem Neuigkeitswert von Ergebnissen fragen, sondern auch der Frage nach ihrer Reproduzierbarkeit mehr Aufmerksamkeit schenken. Das ist ein Paradigmenwechsel und lässt sich nicht gleich umsetzen, aber viele Einrichtungen haben schon damit begonnen, die Zahl der Meldungen sukzessive zu reduzieren und die freiwerdenden Ressourcen stärker in die Bündelung und Einordnung von Themen zu investieren.

\section{Relevanz aus Empfängerperspektive}

Laut einer Studie von 3M („State of Science Index Survey“), die 2018 in 14 Ländern - darunter auch in Deutschland - durchgeführt wurde, denken zwei Drittel der Befragten selten bis gar nicht darüber nach, welchen Einfluss Wissenschaft auf ihr tägliches Leben hat. 63 Prozent sehen zwar eine sehr große Bedeutung der Wissenschaft für die Gesellschaft, aber nur etwas weniger als die Hälfte der Befragten für das tägliche Leben (3M 2018, S. 24, 26). Dabei greifen Menschen im Alltag ständig auf wissenschaftliches Wissen zurück: Wie wird das Wetter und was ziehe ich an? Wie sicher sind unsere Lebensmittel und was kann ich noch kaufen? Soll ich mich impfen lassen, bevor ich in die Tropen reise? Nehme ich die Pille oder gibt es Alternativen? Wenn die Studie recht hat und sich so viele Menschen des Einflusses der Wissenschaft auf ihr tägliches Leben noch immer nicht bewusst sind, dann gibt es für die Wissenschaftskommunikation tatsächlich noch einiges zu tun, denn ein kompetenter Umgang mit wissenschaftlichen Erkenntnissen ist für jede bzw. jeden eine Notwendigkeit.

Prognosen für die Zukunft bleiben schwierig. Und die Klimaforschung ist nur ein Beispiel, das zeigt, dass Wissenschaft auch Gefahr laufen kann, instrumentalisiert zu werden. Das darf Wissenschaftlerinnen und Wissenschaftler aber nicht davon abhalten, sich mit ihrem Expertenwissen aktiv einzubringen. Als Lennart Bengtsson, Hartmut Graßl und Klaus Hasselmann vom Max-Planck-Institut für Meteorologie 1998 den Deutschen Umweltpreis erhielten, lobte der damalige Generalsekretär der Deutschen Bundesstiftung Umwelt die Forscher: „Sie haben durch ihre Arbeit nicht nur wissenschaftlich belegt, daß Menschen mit sehr hoher Wahrscheinlichkeit durch ihr Handeln das Klima weltweit verändern. Sie haben den schwe- 
ren, aber wichtigen Schritt getan, dieses Wissen auch aktiv zu verbreiten und den Menschen so ihre Verantwortung bewußt zu machen." (DBU 1998). Wissenschaft erweitert unsere Handlungsspielräume; sie stellt ein Repertoire an Möglichkeiten bereit. Wie wir mit diesem Wissen umgehen wollen, das muss gesellschaftlich ausgehandelt werden.

Aber nicht jedes Wissenschaftsthema - und sei es noch so spannend ist gleich ein „public issue“. Google Trends liefert gute Beispiele dafür. Die Funktion stellt die Anzahl von tatsächlich eingegebenen Suchbegriffen in einem zeitlichen Verlauf dar und setzt diese in Relation zum gesamten Suchvolumen. Sie ermöglicht, verschiedene Suchbegriffe miteinander zu vergleichen, und ist damit quasi ein Indikator für Relevanz aus Publikumsperspektive. So besaß der Suchbegriff „Gravitationswellen“ bis zu dem Tag, als ihr erstmaliger Nachweis publiziert wurde und ein gigantisches Presseecho weltweit auslöste, keine große Relevanz. Die Auswertung liefert mehr oder weniger eine Gerade mit einem erkennbaren Peak im Februar 2016, danach verschwindet das Interesse sofort wieder. Vollkommen anders sieht das für den Suchbegriff „CRISPR Cas“ aus. Die entscheidenden Publikationen zu CRISPR Cas stammen aus den Jahren 2012/2013. Seitdem klettert die Kurve der Suchanfragen beharrlich nach oben, das Interesse an dem Thema wächst entsprechend der Relevanz, die CRISPR Cas inzwischen hat.

Wissenschaftskommunikation muss die Überbrückung der immer größer werdenden Distanz zwischen Wissenschaft und Gesellschaft zum Ziel haben. Der Präsident der DFG, Peter Strohschneider, spricht von „einer Art kognitiver Unnahbarkeit" der Wissenschaft und konstatiert dramatische Vermittlungsprobleme: Der Abstand zwischen dem, was sich noch vermitteln ließe, und den Grenzen der Wissenschaft wachse ständig (Wissenschaft kommuniziert 7. Februar 2017). Weil Wissenschaft jede Vereinfachung zuwider ist, übersieht sie oft die Vielfalt an Möglichkeiten zur Wissensvermittlung.

Im Rahmen ihrer \#wonachsuchstdu-Kampagne im Sommer 2018 hat die Max-Planck-Gesellschaft Bürgerinnen und Bürger online aufgefordert, Fragen mit Bezug zur Forschung an den Max-Planck-Instituten einzureichen. Über 250 Fragen sind eingegangen, wie zum Beispiel: Gibt es in Europa Sozialtourismus? Was bringen Gehirnjogging-Spiele am Computer? Warum wird die Gesellschaft von heute atheistisch? Warum beklagen sich viele über das Rentensystem? Wie viele Patente stecken in meinem Handy? Warum brauchen wir Länder und Grenzen? Kommt es zu Tierwanderungen wegen der Klimaerwärmung in Deutschland? Und so weiter. Sie spiegeln das Interesse der Menschen wider und zeigen, wo Wissenschaft anknüpfen 
und ins Gespräch kommen kann. Wissenschaft muss von der Sender- in die Empfängerperspektive wechseln!

\section{Verantwortung von Wissenschaft}

Das Vertrauen in Wissenschaft und Forschung ist in Deutschland, folgt man dem von Wissenschaft im Dialog (WiD) erhobenen Wissenschaftsbarometer, 2018 im Vergleich zum Vorjahr leicht gestiegen: Die Zahl derjenigen, die angeben, dass sie in Wissenschaft und Forschung vertrauen, liegt bei 54 Prozent (2017: 50 Prozent). 39 Prozent zeigen sich unentschieden, 7 Prozent (2017: 12 Prozent) sagen, dass sie nicht oder eher nicht in Wissenschaft und Forschung vertrauen (WiD 2018, S. 15 f.).

Ist das Glas also halb voll oder halb leer, wenn es um die Bewertung dieser Ergebnisse geht? Darüber lässt sich trefflich streiten und auch die beiden Autorinnen waren sich in diesem Punkt nicht einig. Unstrittig aber ist, dass Wissenschaft Vertrauen braucht. Deshalb geben die folgenden Zahlen zu denken: Nur 40 Prozent der Befragten und damit weniger als die Hälfte sind der Auffassung, dass Wissenschaftler tatsächlich zum Wohl der Gesellschaft forschten, 46 Prozent sind in dieser Frage unentschlossen (WiD 2018, S. $21 \mathrm{f}$.$) .$

Das Vertrauen in und die Unabhängigkeit von Wissenschaft sind Werte, die bewahrt werden müssen. Von den Forschenden, den Medien, der Wissenschaftskommunikation und der Politik. „Werte bewahren“" schreibt sich immer so leicht - ist aber harte Arbeit. Gute Wissenschaft muss sich dabei in dem Spannungsfeld zwischen Selbstbestimmung (Wissenschaftsfreiheit) und Einbettung in die Gesellschaft finden und bewähren. Vor diesem Hintergrund ist es unverzichtbar, Transparenz über Forschungsvorhaben und ihre möglichen Folgen herzustellen. Die Selbstvergewisserung der Wissenschaft über die „Leitplanken“ ihres wissenschaftlichen Handelns ist eine wesentliche vertrauensbildende Maßnahme und somit ein hilfreiches Instrument in der Wissenschaftskommunikation.

Hier ein Beispiel: Tierversuche sind ein hochsensibles Thema. Sie können nur auf der Basis großen Vertrauens der Gesellschaft in die verantwortlichen Institutionen durchgeführt werden. Die Max-Planck-Gesellschaft hat daher im November 2016 eine Grundsatzerklärung zu Tierversuchen in der Grundlagenforschung abgegeben, in der sie die Unverzichtbarkeit von Tierversuchen betont, sich aber auch zur besonderen Verantwortung des einzelnen Wissenschaftlers für die Versuchstiere bekennt und den mit Untersuchungen an Lebewesen verbundenen ethischen Problemen. Sie hat das 
dem Tierschutz in der Forschung zugrunde liegende 3R-Prinzip („Reduce, Refine, Replace“ - „Vermindern, Verbessern, Vermeiden“) um ein viertes R für „Responsibility“ (Verantwortung) erweitert und Schulungen zur Tierethik eingeführt. ${ }^{1}$ Das Papier wurde auf der Basis umfangreicher Beratungen durch Forscherinnen und Forscher aus verschiedenen Bereichen der Lebenswissenschaften, aus der Verhaltensforschung, Ethik und Kommunikation sowie aus der Forschungspolitik entwickelt.

Auch die Universität Münster hat im Oktober 2017 ein „Leitbild zum ethischen Umgang mit Tieren in der wissenschaftlichen Forschung und Lehre $^{\text {"2 }}$ veröffentlicht, das nun Teil der Regeln guter wissenschaftlicher Praxis an der Universität ist und zusätzlich zu den umfangreichen gesetzlichen Vorgaben als ethische Orientierung für Wissenschaftler dienen soll.

Insgesamt setzen sich die Hochschulen in Deutschland verstärkt mit Verantwortung und Transparenz in der Wissenschaft auseinander. Neben dem Umgang mit Tierversuchen zählen hierzu Themen wie die Drittmittelförderung aus der Industrie, Dual-Use-Forschung oder Nebentätigkeiten von Professorinnen und Professoren. Seit Anfang des Jahres 2019 erarbeitet die Projektgruppe „Transparenz“ als Teil der „Initiative Qualität von Hochschulkommunikation" des Bundesverbands Hochschulkommunikation (siehe unten) an entsprechenden Empfehlungen und Services für Hochschulen.

\section{Verantwortung von Wissenschaftskommunikation}

In dem viel zitierten Wettbewerb, in dem Hochschulen stehen, ist Erfolg nicht mehr allein von Kreativität und wissenschaftlicher Leistung abhängig, sondern zunehmend auch von der Wahrnehmung dieser Leistung und dem Vertrauen, das der Institution entgegengebracht wird. Wissenschaftseinrichtungen muss es daher gelingen, mit ihrer Kommunikation Vertrauen zu schaffen, authentisch zu kommunizieren und - an geeigneten Stellen - Partizipation zu ermöglichen.

Der Wissenschaftsjournalismus als kritischer Beobachter, als bewertende und einordnende Instanz ist schwächer geworden; seine Rolle als Gate

\footnotetext{
${ }^{1}$ Mehr zur Grundsatzerklärung unter: https://www.mpg.de/tierversuche-whitepaper.

${ }^{2}$ Herunterladbar unter: https://www.uni-muenster.de/imperia/md/content/wwu/wwu_leitbild_tierversuche.pdf.
} 
Keeper hat er bereits weitgehend verloren. Dank neuer Kommunikationskanäle erreichen viele Botschaften die Zielgruppen nunmehr direkt. Durch diese veränderten Rahmenbedingungen ist die Verantwortung der Wissenschaftskommunikation, und damit auch die der Hochschulen und Forschungseinrichtungen, gestiegen. Hochschulen und Wissenschaftseinrichtungen sollten in der Kommunikation ihren weitgehend auf die eigene Institution fokussierten Blick erweitern und Themen auch institutionenübergreifend bearbeiten - was in der Wissenschaft selbst eigentlich schon lange üblich ist (der fach- und institutionenübergreifende Austausch und die Zusammenarbeit), sollte auch in der Wissenschaftskommunikation stattfinden. Nur so kann ein wissenschaftlicher Diskurs den Themen angemessen abgebildet werden.

Bereits im Jahr 2013 formulierte der vom Bundesverband Hochschulkommunikation gemeinsam mit Wissenschaft im Dialog (WiD) initiierte Siggener Kreis Werte der Wissenschaftskommunikation, die für die Hochschulkommunikation ebenso wie für andere Bereiche der Wissenschaftskommunikation gelten sollen: Wahrhaftigkeit und Glaubwürdigkeit, Nutzen für die Gesellschaft, Transparenz, Offenheit der Wissenschaft für den aktiven Dialog mit der Gesellschaft, Selbstkritik und Veränderungsbereitschaft, Unabhängigkeit sowie die Kooperationsbereitschaft aller Akteure (Siggener Kreis 2013). Diese Werte, die ebenso Werte der Wissenschaft selbst sind, helfen dabei, der Verantwortung gerecht zu werden.

Unter dem Stichwort Qualität in der Hochschulkommunikation sind beispielsweise neben der seit 1998 bestehenden „Initiative Qualität von Hochschulkommunikation" (IQ_HKom) des Bundesverbands Hochschulkommunikation die aus dem Siggener Kreis hervorgegangenen „Leitlinien zur guten Wissenschafts-PR"3 zu nennen. Ziel der federführenden Organisationen bei der Entwicklung dieser Leitlinien war, so viele Akteure wie möglich zu beteiligen und damit die vielfältigen Initiativen zur Wissenschaftskommunikation der vergangenen Jahre zu bündeln - auch um dadurch mehr Durchsetzung und Akzeptanz der Leitlinien zu erreichen. Die außeruniversitären Forschungseinrichtungen und Wissenschaftsakademien haben diese Leitlinien - teilweise in leicht modifizierter Form - weitgehend übernommen.

${ }^{3}$ Herunterladbar unter: https://www.bundesverband-hochschulkommunikation.de/fileadmin/user_ upload/IQ/IQ_Leitlinien/Leitlinien-gute-Wissenschafts-PR_final.pdf. 


\section{Literatur}

Altbach, P., \& de Wit, H. (7. September 2018). Too much academic research is being published. University Word News. https://www.universityworldnews.com/ post.php?story $=20180905095203579$. Zugegriffen: 24. März 2019.

DBU. (1998). Chancen für globalen Klimaschutz durch Forschungsarbeit stark erhöht. Pressemitteilung vom 25. September 1998. https://www.dbu.de/123artikel25161_2442.html. Zugegriffen: 19. März 2019.

Edelman Trust Barometer. (2018). Executive summary. https://www.edelman.de/newsroom/studien-insights/edelman-trust-barometer-2018/. Zugegriffen: 19. März 2019.

Leßmöllmann, A., Hauser, C., \& Schwetje, T. (2017). Hochschulkommunikation erforschen. Hochschulkommunikatoren als Akteure: Ergebnisse einer Online-Befragung - 1. Welle. Karlsruher Institut für Technologie KIT. https:// www.geistsoz.kit.edu/germanistik/downloads/Zwischenbericht $\% 20$ Hochschulkommunikation\%20erforschen\%201.\%20Welle\%20Leßmöllmann\%20 Hauser\%20Schwetje.pdf. Zugegriffen: 19. März 2019.

Schnabel, U., \& Spiewak, M. (2. August 2018). Die Ungewissheit umarmen. Interview mit Helga Nowotny. DIE ZEIT, 32. https://www.zeit.de/2018/32/helga-nowotny-ungewissheit-soziologie-forschung/komplettansicht. Zugegriffen: 19. März 2019.

Schwetje, T., Hauser, C., \& Leßmöllmann, A. (2017). Hochschulkommunikation erforschen. Hochschulkommunikatoren als Akteure: Ergebnisse einer Online-Befragung - 2. Welle. Karlsruher Institut für Technologie KIT. https://www.geistsoz.kit. edu/germanistik/downloads/Projektbericht-Hochschulkommunikation-erforschen-2. Welle-Schwetje-Hauser-Lessmoellmann.pdf. Zugegriffen: 19. März 2019.

Schrader, C. (3. September 2018). Und nun zum Klimawandel. Süddeutsche Zeitung. https://www.sueddeutsche.de/wissen/umwelt-der-taegliche-klimawandel-1.4114901. Zugegriffen: 19. März 2019.

Siggener Kreis. (2013). Siggener Denkanstoß. https://www.wissenschaft-im-dialog. de/projekte/siggener-kreis/. Zugegriffen: 19. März 2019.

Wissenschaft im Dialog (WiD). (2018). Wissenschaftsbarometer 2018. https:// www.wissenschaft-im-dialog.de/projekte/wissenschaftsbarometer/wissenschaftsbarometer-2018/. Zugegriffen: 19. März 2019.

Wissenschaft kommuniziert. (7. Februar 2017). „Wir haben dramatische Vermittlungsprobleme“. Teil 2 des „Wissenschaft kommuniziert“-Gesprächs mit DFG-Präsident Prof. Dr. Peter Strohschneider. https://wissenschaftkommuniziert.wordpress.com/2017/02/07/wir-haben-dramatische-vermittlungsprobleme/. Zugegriffen: 19. März 2019.

3M. (2018). State of science index 2018. Global report. https://multimedia.3m. $\mathrm{com} / \mathrm{mws} / \mathrm{media} / 1515295 \mathrm{O} /$ presentation-3m-state-of-science-index-2018-global-report-pdf.pdf. Zugegriffen: 24. März 2019. 
Dr. Christina Beck ist seit 2008 Leiterin der Kommunikation der Max-Planck-Gesellschaft (MPG). Zuvor war sie zehn Jahre lang als Redakteurin verantwortlich für die biologisch-medizinische Berichterstattung der MPG und hat dabei auch das Schüler-Lehrer-Portal www.maxwissen.de sowie die MAX-Hefte aus der Taufe gehoben. 2016 war sie Forschungssprecherin des Jahres, 2018 erhielt sie den Publikumspreis als beliebteste Forschungssprecherin. Seit 2016 ist sie Mitglied der Steuerungsgruppe der Allianzinitiative Tierversuche verstehen; in 2018 war sie als Kommunikationsexpertin zum Workshop Wissenschaftskommunikation des BMBF geladen.

Julia Wandt ist seit 2010 Leiterin der Stabsstelle Kommunikation und Marketing sowie Pressesprecherin der Universität Konstanz. Seit 2014 ist die Medien- und Kommunikationswissenschaftlerin zudem Vorsitzende des Bundesverbands Hochschulkommunikation - dem Zusammenschluss der Kommunikationsverantwortlichen an Hochschulen in Deutschland. Darüber hinaus ist sie regelmäßig in Projektgruppen und Anhörungen zu Status quo und Zukunft von Wissenschaftskommunikation und Transfer aktiv, wie zum Beispiel des BMBF und des Deutschen Bundestages.

Open Access Dieses Kapitel wird unter der Creative Commons Namensnennung 4.0 International Lizenz (http://creativecommons.org/licenses/by/4.0/deed.de) veröffentlicht, welche die Nutzung, Vervielfältigung, Bearbeitung, Verbreitung und Wiedergabe in jeglichem Medium und Format erlaubt, sofern Sie den/die ursprünglichen Autor(en) und die Quelle ordnungsgemäß nennen, einen Link zur Creative Commons Lizenz beifügen und angeben, ob Änderungen vorgenommen wurden.

Die in diesem Kapitel enthaltenen Bilder und sonstiges Drittmaterial unterliegen ebenfalls der genannten Creative Commons Lizenz, sofern sich aus der Abbildungslegende nichts anderes ergibt. Sofern das betreffende Material nicht unter der genannten Creative Commons Lizenz steht und die betreffende Handlung nicht nach gesetzlichen Vorschriften erlaubt ist, ist für die oben aufgeführten Weiterverwendungen des Materials die Einwilligung des jeweiligen Rechteinhabers einzuholen. 